\title{
Organização dos serviços de saúde e a gestão do cuidado à tuberculose
}

\section{Organization of health services and tuberculosis care management}

\author{
Anne Jaquelyne Roque Barrêto ${ }^{1}$ \\ Lenilde Duarte de Sá ${ }^{2}$ \\ Jordana deAImeida N ogueira ${ }^{3}$ \\ Pedro Fredemir Palha ${ }^{4}$ \\ Patrícia Geórgia de O liveira Diniz Pinheiro ${ }^{5}$ \\ Nilma M aria Porto de Farias ${ }^{6}$ \\ Débora Cezar de Souza Rodrigues ${ }^{5}$ \\ Tereza Cristina Scatena Villa ${ }^{4}$
}

${ }^{1}$ Faculdade de Enfermagem Nova Esperança. Rua Dílson Pessoa 69, Água Fria. 58077-290 João Pessoa PB. annejaque@gmail.com ${ }^{2}$ Departamento de Enfermagem em Saúde Pública e Psiquiatria, UniversidadeFederal da Paraíba.

${ }^{3}$ Departamento de Enfermagem M édicoCirúrgica, Universidade Federal da Paraíba.

${ }^{4}$ Departamento de Enfermagem M aternoInfantil eSaúde Pública, Escola de Enfermagem de Ribeirão Preto, Universidade de São Paulo. ${ }^{5}$ Universidade Federal da Paraíba

${ }^{6}$ Complexo Hospitalar de Doenças Infecto-Contagiosas Dr. Clementino Fraga, Secretaria Estadual de Saúde daParaíba.
Abstract The scope of this study was to analyze the discourse of managers regarding the relationship between the organization of the health services and tuberculosis care management in a city in themetropolitan region of J oão Pessoa, State of Pernambuco. Using qualitative research in the analytical field of the French line of Discourse Analysis, 16 health workers who worked as members of the management teams took part in the study. The transcribed testimonials were organized using Atlas.ti version 6.0 software. After detailed reading of the empirical material, an attempt was madeto identify the paraphrasic, polyssemic and metaphoric processes in the discourses, which enabled identification of the following discourse formation: Organization of the health services and the relation with TB care management: theory and practice. In the discourse of the managers the fragmentation of the actions of control of tuberculosis, the lack of articulation between the services and sectors, the compliance of the specific activities for TB, as well as the lack of strategic planning for management of care of the disease are clearly reveal ed. In this respect, for the organization of the health services to be effective, it is necessary that tuberculosis be considered a priority and acknowledged as a social problem in the management agenda.

Key words Nursing, Health management, Tuberculosis
Resumo Esteestudo tevecomo objetivo analisar a discursividade de gestores sobre a relação entre a organização dos serviços de saúde e a gestão do cuidado à tuberculose (TB) em um município da região metropolitana de João Pessoa/PB. Conduzido pela pesquisa qualitativa no campo analítico da Análise de Discurso de linha francesa, participaram 16 trabalhadores de saúde que atuavam como integrantes de equipes gestoras. Os depoimentos transcritos foram organizados com uso do software Atlas.ti versão 6.0. A pós leitura minuciosa do material empírico procurou-se observar nos discursos os processos parafrásicos, poli ssêmicos e metafóricos, os quais possibilitaram a identificação da seguinte formação discursiva: organização dos serviços de saúde e a relação com a gestão do cuidado à TB; o plano e a prática. Nos discursos dos gestores evidencia-sea fragmentação das ações de controle da tuberculose, a falta de articulação entre os serviços e os setores, o cumprimento de atividades espećficas à $T B$, bem como a falta de planejamento estratégico para gestão do cuidado da doença. N esse sentido, para que a organização dos serviços de saúde seja efetiva, se faz necessário que a tuberculose seja prioridade na agenda da gestão e reconhecida como um problema social. Palavras-chave Enfermagem, Gestão em saúde, Tuberculose 


\section{Introdução}

Este estudo teve como objeto de análisea discursividade de gestores sobre a relação entre a organização dos serviços de saúde e a gestão do cuidado à tuberculose (TB) em um município da região metropolitana de João Pessoa/PB. Teoricamente encontra-se ancorado no conceito de Gestão do Cuidado.

Uma vez que o Brasil é considerado o 19ạ no número de casos de TB no mundo, faz-se necessário incrementar ações e serviços que reduzam a morbimortalidade relacionada à doença no país. É importante reconhecer que o Programa Nacional de Controle da Tuberculose (PNCT) tem definido prioridades que, na perspectiva da concepção renovada da Atenção Primária deSaú$\mathrm{de}^{1}$, estas estão na agen da da Política Nacional de Atenção Básica em Saúde, cuja maior expressão tem sido a Estratégia Saúde da Família (ESF).

$\mathrm{Na}$ busca de fortalecer a Atenção Primária em Saúde (APS) objetivaram aumentar o poder no primeiro nível de atenção enquanto coordenador de outros níveis, com vista de garantir um cuidado integral e humanizado no sistema e serviços de saúde por meio de estratégias de mercado que garantam o papel de porta de entrada e de acesso à atenção especializada com base em mecanismos de coordenação hierárquica ${ }^{1}$. Essa possibilidade se concretiza quando os serviços reconhecem no território um espaço de intervenção contínua, o que facilita o estabelecimento de vínculo e responsabilidade entre a coordenação, a equipe de saúde e a população local.

O PNCT admite a importância de horizontalizar o controle da TB, estendendo-o para todos os serviços do SistemaÚ nico de Saúde(SUS). Portanto, visa a integração da gestão do cuidado da doença com a APS, incluindo o Programa de Agentes Comunitários de Saúde (PACS) e a ESF para garantir a efetiva ampliação do acesso ao diagnóstico e ao tratamento ${ }^{2}$. No entanto, assim como o abandono do tratamento, 0 atraso no diagnóstico é considerado um dos grandes desafios que deve ser enfrentado pela gestão e trabaIhadores de saúde.

O problema do atraso do diagnóstico está muitas vezes relacionado ao despreparo das equipes de saúde para identificar sintomáticos respiratórios e à demora em confirmar o diagnósti$\mathrm{CO}^{3}$. N esse sentido, o modo de conduzir os processos de gestão do cuidado pode fortalecer ou fragilizar a organização dos serviços voltados à atenção às pessoas doentes ou expostas à TB. Por gestão do cuidado enten de-se a forma como o "cuidar se revela e se organiza na interação propiciada entre sujeitos, podendo gerar interações e subversões potencialmente emancipatórias ou restritivas das múltiplas liberdades humanas"4.

A gestão do cuidado comporta, pelo menos, três dimensões: profissional, organizacional esistêmica. Na dimensão profissional é onde ocorre o singular encontro entre trabalhador-usuário cuja finalidade denota na resolução das necessidades elencada pelo usuário ${ }^{5}$. A dimensão sistê mica refere-se ao conjunto de serviços de saúde, com suas diferentes funções e graus de incorporação tecnológica, bem como os fluxos estabelecidos entre si. Estes por sua vez, serão definidos por protocolos e regulados no nível central com o objetivo de garantir acesso dos usuários às tecnologias de cuidado que necessitam de complexas redes de cuidado institucionais 5 .

A dimensão organizacional, adotada nesteestudo, é compreendida como um espaço de desdobramentos da divisão técnica e social do trabaIho em saúde, com todas as "implicações para complexos processos coordenação de múltiplas práticas profissionais ou das múltiplas gestões profissionais do cuidado" 5 . Ressalta-se que é neste espaço onde questões relativas ao registro e ao uso da informação, à criação de espaços de diálogo, ao estabelecimento de fluxos de usuários nos serviços de saúde, à normatização de processos de trabalho, a corresponsabilização dos atores envolvidos, entre outros aspectos, vão configurar uma nova lógica em que a responsabilidade da gestão e a conformação da dinâmica de relacionamento da equipe ocuparão lugar central ${ }^{5}$.

Um ator importante na gestão do cuidado em sua dimensão organizacional éo profissional que atua como gestor ou que desenvolve ações gerenciais. Neste estudo, foi considerado como gestor 0 apoiador matricial (AM) concebido como um dinamizador do processo de reformulação das práticas de saúde que visa o estabelecimento devínculo, acolhimento eresponsabilização das equipes de saúde para construção do cuidado integral nos espaços da micropolítica ${ }^{6}$. A escolha do AM como sujeito desse estudo foi intencional, tendo em vista ser este um ator cuja subjetividade pode auxiliar na articulação entre Unidade de Saúde da Família (USF) e Distrito Sanitário (DS) e, consequentemente, na compreensão da organização dos serviços do sistema de saúde municipal para gestão do cuidado à TB.

O AM possibilita a introdução, no processo detrabal ho, de novas possibilidades de trocas de saberes entre os diversos profissionais, favorecendo uma maior articulação e qualificação da 
rede de serviços que compõe o sistema de saúde. Nesse sentido, cabe questionar: o que apresenta a discursividade dos AM sobre a relação entre a organização dos serviços e a gestão do cuidado à TB considerando ser a ESF, pela política de saúde local, a principal porta de entrada no sistema de saúde no município estudado?

Este artigo objetiva analisar a discursividade de gestores sobre a relação entre a organização dos serviços de saúde ea gestão do cuidado à TB em um município da região metropolitana de João Pessoa (PB), Brasil.

\section{M étodo}

Este estudo foi conduzido pela pesquisa qualitativa no campo analítico da análise de discurso (AD) de linha francesa ${ }^{7}$. Busca-se na abordagem qualitativa um suporte para operacionalizar as inferências elencadas neste tipo de pesquisa, já que ela envolve mais do que a expressão de um fenômeno singular?.

No processo de análise do material empírico foi utilizada a técnica de $A D^{7}$, uma vez queéindicada nas pesquisas qualitativas pelas possibilidades de relacionamento dos materiais que envolvem valores, juízos necessários e preferíveis como argumentos ou como meios capazes de revelar a visão de mundo e, portanto, a posição ideológica do sujeito discursivo.

Elegeu-se como cenário do estudo um município da região metropolitana deJ oão Pessoa (PB), considerado prioritário pelo M inistério da Saúde (MS) para o controle da TB. Atualmente, nesse município, as ações de controle da tuberculose são realizadas pelas equipes de Saúde da Família (SF), Centros Especializados, hospitais de referência e laboratórios que realizam a baciloscopia. Dentre as USF $24,5 \%$ adotaram o Tratamento Diretamente Observado (TDO) como estratégia de controle dos casos. Convém ressaltar que a maior parte dos casos residentes no município é diagnosticada pelo ambulatório de pneumologia do Hospital de Referência, unidade de referência estadual para diagnóstico e tratamento de doenças transmissíveis, considerado ainda a principal porta deentrada - preferida pelos usuários - para o tratamento da tuberculose ${ }^{8}$.

Os sujeitos participantes foram 16 trabal hadores de saúde, AM, que atuavam como integrantes de equipes gestoras responsáveis pela organização dos serviços de saúde, na articulação entreas ESF eos DS. No município estudado o AM foi inserido em $2006 \mathrm{com}$ a finalidade de ser um facilitador na implantação do Plano Municipal deSaúde, principalmentedo fortalecimento da ESF como porta de entrada do sistema.

Os critérios de inclusão dos sujeitos considerados foram: atuar como AM no sistema local de saúdee ser domiciliado no município escolhido para a realização da pesquisa. Os profissionais colaboradores estão identificados pela letra $\mathrm{G}$ (gestor) dispostos na sequência de G1 a G16.

A coleta de informações foi realizada nos meses de agosto esetembro do ano de 2009. Depois de concordarem em participar do estudo, os AM assinaram o TCLE. As entrevistas foram feitas no local de trabal ho dos sujeitos, gravadas individualmente, tendo sido utilizado aparelho audio-portátil e M P4.

Inicialmente foram realizadas leituras exaustivas do material empírico, adquirido a partir dos depoimentos dos sujeitos, com a finalidade de identificar sinais, vestígios e pistas. Realizado este processo foi constituído o corpus discursivo. A partir deste foram observados os enunciados em relação à organização dos serviços e à gestão do cuidado à TB.

$\mathrm{Na}$ codificação do corpus discursivo foi empregado software Atlas.ti versão 6.0. Com o uso dessa ferramenta foram criados cinco códigos: Descentralização do controle da TB para APS; Cuidado eresponsabilização da equipeSF com 0 doente de TB; A compreensão do DOTS (Directly Observed Treatment Short Course) como estratégia de controle da TB; Passividade das equipes saúde na produção e análise das informações acerca da TB e Planejamento das ações de controle da TB. A pós a codificação das 16 entrevistas, foram gerados relatórios, sobre os quais foi aplicada a técnica de análise.

$\mathrm{Na}$ segunda etapa da análise procurou-se observar nos discursos os processos parafrásicos, polissêmicos e metafóricos, os quais possibilitaram a identificação da seguinte formação discursiva: Organização dos serviços de saúde e a relação com a gestão do cuidado à TB - o plano ea prática.

Em seguida os enunciados que caracterizavam os discursos dos sujeitos foram analisados quanto à posição ideológica do sujeito, sua relação com outros discursos, redes de filiações históricas, interdiscurso e à memória discursiva. Procurou-se identificar as contradições entre o que se encontra estabelecido no plano de saúdee a posição do sujeito em relação à gestão do cuidado à TB.

0 projeto de pesquisa foi enviado ao Comitê de Ética do Centro de Ciências da Saúde da Uni- 
versidadeFederal da Paraíba (CCS/UFPB), atendendo às orientações éticas e legais, inerentes ao protocolo de pesquisa envolvendo seres humanos, contido na Resolução 196/96 CNS.

\section{Resultados ediscussão}

Os discursos dos gestores mostram que a gestão municipal estabelecea ESF como eixo norteador da organização dos serviços, de modo a garantir 0 acesso às ações e serviços ofertados no âmbito da APS ${ }^{4}$. N esse sentido a gestão municipal, através da área técnica da $T B$, tem articulado junto ao Centro de Referência para tratamento da doença, um reordenamento no fluxo deatendimento à pessoa suspeita e/ou doente de TB. Essa iniciativa se faz necessária porque, além de cumprir com as diretrizes organizacionais do SUS, mostra o reconhecimento da TB como área de responsabilidade da APS, conforme o plano municipal de 2006 e do Pacto (2006):

A tualmente está passando por um processo de descentralização. I nicialmente todos os pacientes estavam concentrados no [H ospital de Referência], eagora [...] estes pacientes estão sendo encaminhados para a própria U nidade de Saúde, onde eles vão ser acompanhados pelo médico da unidade. (G1)

Segundo os AM , embora exista oferta deconsultas, exames, medicamentos e o benefício da alimentação, há relutância do doente de TB em procurar a USF para o diagnóstico da doença. Há, também, resistências por profissionais de saúde que contraria a política de descentralização das ações de controle da TB para as USF, uma vez que continuam a encaminhar os casos suspeitos para o centro de referência de TB.

Elas [pessoas da comunidade] não querem ser atendidas no serviço [...]. Estão [as pessoas doentes de TB] sendo induzidas a ir para o [H ospital de Referência] e a gente tem que entender e saber quea responsabilidadenão édo [H ospital de Referência] e sim da Atenção Básica. M as é isso que eu falei, éa questão da preparação mesmo dos profissionais em acolher esse tipo de usuário. (G5)

Culturalmente as pessoas têm a visão que lá [H ospital de Referência] élocal que se trata a tuberculose, então elas mesmas vão pra lá. (G2)

A descentralização no contexto do setor saúde é uma diretriz importante para o desencadeamento de ações voltadas a garantir 0 acesso às ações de saúde por inúmeros sujeitos, antes excluídos dessa assistência?. Estudo realizado sobre a implementação da estratégia SF em grandescen- tros urbanos no Brasil aponta avanços da descentralização das ações e programas de saúde pública. Porém, informa que em um dos municípios o cuidado à tuberculose foi considerado uma das ações mais difíceis de ser descentralizada ${ }^{1}$.

Percebe-se também nos fragmentos que os gestores reconhecem à importância da atenção à pessoa doente de TB ser realizada pela equipeSF. Vale salientar, que o hospital de referência, nas últimas cinco décadas, foi a principal porta de entrada para atenção a TB, além de ter sido o primeiro serviço a implantar o TDO no estado. Nesse sentido, uma das dificuldades enfrentadas pelo gestor relaciona-se no reconhecimento da ESF pela população, como local de diagnóstico e tratamento para a doença.

A dificuldade de consolidar a ESF como porta deentrada preferencial ecentro ordenador eintegrador das redes de serviços, também foi identificada em outros municípios no Brasil ${ }^{1}$. Com o objetivo de superar o histórico insulamento das ações de atenção básica, municípios adotaram estratégias para integrar o SF com a rede deserviços regionais, visando, sobretudo, a garantia do acesso e ações resolutivas, a criação de sistemas de informação e regulação, de serviços especializados, apoio matricial e fóruns de discussão ${ }^{10}$.

Por sua vez, a descentralização das ações de controle da TB para a ESF necessita do envolvimento de distintos atores, de acordo com as responsabilidades inerentes à função de cada um, de modo a operacionalizar ea desenvolver ações estratégicas que visem à eficiência e eficácia da ESF no controle da TB, principalmente relacionadas ao diagnóstico precoce e à conclusão do tratamento ${ }^{11}$.

O AM ao atuar na organização do trabalho entre as ESF e os DS, é ator importante para o desenvolvimento de práticas que estimulem a capacidade de produção de saberes - em ato -, de modo a promover a ampliação de habilidades para análise e da ação dos trabal hadores no sentido de coprodução de saúde e de autonomia de sujeitos ${ }^{12}$. Contraditoriamente, nos discursos dos sujeitos, não se observam enunciados que evidenciem movimentos nessa direção e nem que favoreçam a efetiva descentralização do cuidado à TB para ESF.

Chama atenção nos discursos a valorização, dada pelo AM, do acesso a exames e tratamento gratuito. No corpus discursivo não foram encontrados fragmentos que versem sobre a vulnerabilidade social, fenômeno geralmente associado ao doente de TB e que poderia desencadear ou justificar a criação/implementação de ações e 
políticas intersetoriais. Percebe-se, no discurso, que a organização do serviço está marcada pelo cumprimento de protocolos préestabelecidos, monitoramento deindicadores egarantia deacesso as tecnologias duras (realização de procedimentos, exames clínicos, insumos) voltadas ao diagnóstico da doença:

$\mathrm{N}$ a U nidade a gente dispõe da medicação, dispõe do serviço de fazer o exame e do acompanhamento. A gente tem, [...] 0 apoio da questão da alimentação. (G8)

0 controle da tuberculose segue o protocolo do Ministério. Tem o livro de Sintomáticos Respiratórios. Então as equipes fazem o acompanhamento dos Sintomáticos Respiratórios. (G7)

o Distrito manda uma moto, pegar, eaí faz na Unidade todo procedimento. Passa pel o processo de informação, e ai o motoquei ro leva para o laboratório. Quando volta o resultado, aí todo o tratamento (se for confirmado), começa a tomar os medicamentos, acompanhar essa família. (G3)

Reconhece-sea importância de assegurar nos espaços de cuidado instrumentos, equipamentos e insumos que venham garantir o acesso e 0 tratamento para as pessoas com TB. No entanto, não são identificadas, nos discursos dos gestores, enunciados que revelem sensibilidade em relação ao preparo do doente para receber o diagnóstico de TB, detecção precoce dos casos e construção de projetos terapêuticos singulares, aspectos coerentes com a construção de um cuidado que permeia a integralidade e a humanização, defendidos pela política municipal, e que deveria nortear a organização dos serviços.

Observa-se também, nos discursos dos gestores, que há dissonância entre o que defende a política municipal; de construir uma rede deserviços de saúde integral, humanizados e permeados por processo de EPS e de cogestão6; com a prática de gestão. Ossujeitos deslizam parauma concepção quevaloriza a hierarquização descendente e burocratizada de organização dos serviços de saúde, reforçando a racionalização gerencial hegemônica.

A inserção do AM , como estratégia de gestão elencada na política municipal, surgiu com a finalidade de implementar novos arranjos que produzissem outra cultura e outras linhas de subjetivação, não centradas no corporativismo e na alienação do trabalhador - características da racionalização gerencial hegemônica - e sim, na produção de sujeitos autônomos e comprometidos com a construção do cuidado tanto individual quanto coletivo ${ }^{12}$. Esse processo deveráacontecer em função de movimentos coletivos orien- tados pelo conceito de cogestão. A ideia de cogestão fundamenta a compreensão de que a gestão é uma tarefa coletiva e que nos espaços coletivos deve-se cumprir mediante três funções básicas: administrar e planejar processos de trabal ho objetivando a produção de valores de uso; alterar as relações de poder e construir a democracia em instituições e; na capacidade de influir na constituição de sujeitos ${ }^{13}$.

A política municipal de saúde do município cenário do estudo ressalta a utilização de espaços de cogestão como fundamentais para identificação das necessidades de saúde da população e dos processos de decisão $0^{6}$. Vale ressaltar que na perspectiva da construção de um projeto que toma por base uma linha de cuidado integral, é importante reconhecer que este é um processo instituinte e que raramente se tem o pleno controle sobre todas as variáveis que envolvem o desenvolvimento institucional, principalmente aqueles que valorizem processos democráticos, de inclusão e de solidariedade, como informados no plano municipal de saúde 6 .

Outro ponto importante identificado nos enunciados dos gestores, diz respeito à centralização e à hierarquização do processo de informação da área técnica de TB da SM S para as USF. O que se observa é que a equipe de saúde é apenas receptora da informação e não coparticipe do processo de monitoramento e avaliação das informações de TB:

Esse cadastro vai para a Secretaria e tem um pessoal quetem essecontrolelá. Esse pacienteésempre monitorado até a alta, até a cura e as pessoas envolvidas com ele também fazem o tratamento. (G6)

Eles [área técnica da TB] que fazem o controle do saldo, fazem o controle mensal do formulário e mandam para as equipes. $E$ a gentefaz um encontro com eles, geralmente na reunião matricial que é onde trazem os cálculos ea gente tem uma noção dos casos que tem no município [...]. (G4)

Os fragmentos discursivos revelam passividade das equipes de saúde na produção das informações sobre os casos de TB. Esse processo contraria a política municipal no que concernea produção de informações em saúde que, descentralizadas, possam alimentar o processo de planejamento nos espaços micropolíticos. N essesentido, a produção e a avaliação dessas informações deverão ser realizadas pelos atores que trabal ham diretamente na produção dos dados, ou seja, nos serviços de saúde. Também não évisível nos discursos a utilização dessas informações para tomada de decisão junto as equipes de SF. 
No caso da vigilância de TB o conhecimento dos casos da doença que ocorrem na população é fundamental para a adoção de medidas que visam a interrupção da sua transmissão para grupos e pessoas vulneráveis ou não. Entretanto, os casos diagnosticados e notificados pelos serviços desaúde podem representar apenas uma proporção dos casos de TB. A manutenção periódica, atualização e avaliação da base de dados do Sistema de Informação de Agravos de N otificação (SINAN) são condições fundamentais para o acompanhamento da morbimortalidade dos casos de TB. Sua utilização efetiva possibilita a realização do diagnóstico dinâmico da ocorrência do agravo na população, bem como a definição de estratégias de intervenção e avaliação no campo da gestão de serviços e ações em saúde ${ }^{14}$, ou seja, a materialização da gestão do cuidado 5 .

Não se deve desprezar a importância do monitoramento de casos de TB nos diversos níveis de gestão. No entanto, é importante que o processo de construção dessas informações sejam oriundas dos sujeitos que a produzem (trabaIhadores de saúde), inclusive com a participação de representantes da comunidade local.

OsAM ao serem questionados sobreo DOTS revelaram desconhecimento dessa estratégia, associando-a geralmente ao benefício do café da manhã, à entrega da medicação e ao TDO:

Realmente essetratamento na U nidade deSaúde, ele tem sido bastante controlado. Os pacientes estão recebendo essa medicação na U nidade. (G1)

$M$ as a medi cação está sen do encaminhada bem direitinho. [...] eaí esse usuário recebe o DOT, que é uma cesta de alimentação. (G12)

$M$ uito interessante porque realmentea alimentação para o paciente de tuberculose é fundamental. (G10)

A redução da estratégia D OTS pelos gestores paraTD 0 , a entrega de medicamento, ao benefício de cesta básica ou oferta de café da manhã nas USF, fragiliza a construção de um cuidado integral à pessoa doente de TB e fortalece, além da exclusão social, uma organização de serviços e de atenção centrada na cura, o que reforça um modelo de atenção centrado em fundamentos biomédicos.

Ressalta-se que a estratégia DOTS compreende cinco elementos essenciais - 0 envolvimento dos governos, o suporte financeiro com o programa de TB, equipes de trabalho treinadas e interessadas; rede laboratorial com equipamentos e recursos humanos qualificados (pesquisa deBAAR); administrar eassistir engolir os medicamentos (TDO); provisão de medicamentos gratuitos para tratamento; sistemas de informação para monitorar os casos, tratamento, evolução e resultados ${ }^{15}$ - estes fundamentais para a gestão ao cuidado à TB.

O TDO é um elemento chave da estratégia DOTS ${ }^{16}$. É considerada estratégia eficiente para controlar as dificuldades da baixa adesão ao tratamento da tuberculose e investimento para aumentar as taxas de cura e diminuir o abandono ${ }^{15}$. Estudos relacionam o declínio da taxa de abandono e aumento na taxa de cura a implantação do TDO nos serviços de saúde ${ }^{17,18}$, mas esse deve ser operacionalizado em conjunto com os outros pilares do DOTS. Destaca-se que o TDO, enquanto tecnologia de gestão do cuidado para o controle da TB, constitui um conjunto de atividades voltadas para o planejamento do tratamento do doente efamília a ser executada por uma equipe de saúde.

Percebe-seno discurso dosAM queexisteuma preocupação da gestão no que concernea garantia dos equipamentos organizacionais necessários para a execução do TDO pela ESF. Por outro lado, observa-se haver necessidade de se investir no estabelecimento de vínculo profissional e doente de TB, considerando os modos de vida, sua dinâmica familiar, suas crenças, opiniões e conhecimentos a respeito da doença ${ }^{3}$.

Entretanto, há discursos de apoiadores que se aproximam dos conceitos de humanização e integralidade do cuidado quando mencionam que a equipe deSF tem responsabilidade sobre o adoecimento das pessoas em sua área adstrita:

E aí ter essa questão da supervisão, na responsabilização da equipe de saúde da família àquele usuário [...] Não controle no sentido ruim de controlar, mascontroleno sentido desaber queo usuário precisa ser cuidado. [...] Eleé usuário que é de responsabilidade da equipe. $\mathrm{E}$ a partir do momento que implementa isso [TS], a equipe é responsável por esse cuidado. (G8)

M as éum programa [deTuberculose] quevêo usuário como um todo, oferecendo um suporte nutricional e também fazendo esse acompanhamento da equipe com o usuário. E não só medicálo edei xá-lo em casa, a equipe tem queestar acompanhando. (G16)

Nos fragmentos discursivos são revelados conceitos importantes como: cuidado e responsabilização. No setor saúde a prática do cuidado foi constituindo-se como um campo de construção de práticas técnicas cuidadoras, social e historicamente determinada ${ }^{19}$. Cuidar das pessoas é mais do que construir um objeto eintervir sobre ele; é interagir, considerar, reconstruir-se e 
querer construir novos projetos. 0 ato de cuidar também pode ser concebido como forma de conhecer ereinventar cotidianos ${ }^{20}$. Finalmente, "cuidar é uma atitude de responsabilidade. Responsabilizar-seéser capaz de responder, étrazer para si a função da resposta por determinada situação ou ato. [...] 0 ponto central é o fortalecimento dos laços entreo sujeito quebusca $o$ atendimento, o serviço e o território onde deve ser baseado 0 atendimento" 21 .

A responsabilidade dos profissionais de saúde em cuidar das pessoas deve constituir a essência do trabalho em saúde. No ato da produção do cuidado, a responsabilização, se dá na busca contínua da compreensão e resolução das demandas e necessidades do usuário seja no âmbito individual e/ou coletivo. Nesse sentido, a compreensão do processo de cuidar deve alicerçar-se numa relação de compromisso ético-político, de sinceridade, responsabilidade e confiança junto ao outro equeeste processo possa expressar compromisso ético nas relações de gestores e profissionais de saúde; usuários do sistema ${ }^{20,21}$.

Cabe lembrar que a gestão é essencial para disparar estratégias que visem o comprometimento por parte dos profissionais de saúde, neste caso da equipe de SF, na produção do cuidado das pessoas sob sua responsabilidade.

Em relação à gestão do cuidado da TB a história mostra uma prática hegemônica que pouco impacta no controle efetivo da doença. Embora se observe medidas que reforçam mudanças, a forma como estão organizados os serviços e como atuam os profissionais, tendem a retardar esse processo. N esse sentido, o modelo de gestão impedea efetividade, não apenas das ações de controle da TB na APS, mas, principalmente, cria obstáculos à ruptura de práticas de saúde divergentes com modelo de atenção à saúde consoante com os enunciados éticos do M ovimento pela Reforma Sanitária e da consolidação do SUS, que coloca em pauta a saúde como direito de cidadania.

Os discursos dos gestores, no que diz respeito ao planejamento do controle da TB em seu território, mostram que não há planejamento específico. Quando existeédemaneira pontual a partir de novos casos que surgem no serviço de saúdee decaráter normativo. Reconhecem queo controle da TB é importante, mas não constitui prioridade tendo em vista ao número reduzido de casos cadastrados na Unidade de Saúde:

A gente ainda não tem uma ação voltada para o controle da tuberculose [...]. A princípio a gente faz o planejamento a partir das reuniões, se reúne para planejar eai a gente utiliza critério da epidemiologia. (G2)

[...] só para esse planejamento [deTB] não. Até porque os números de casos de tuberculose aqui na [Unidade de Saúde] só são dois no momento. (G5)

$\mathrm{H}$ oje em dia a gente está tentando fazer acompanhamento dos Sintomáticos Respiratórios mensalmente. Que era muito difícil, a gente está tentando planejar o acompanhamento ea busca ativa também de quem abandona o tratamento. Então a gente está tentando fazer mês a mês ol har os livros mesmo. Fazer o exercício, ver se tem sintomático, não teve, o que está fazendo o acompanhamento, a equipe de fato está acompanhando. (G7)

Priorizar as ações nos serviços de saúde, a partir do quantitativo de casos identificados em determinado território, aponta para um processo de organização de serviços que exclui as reais necessidades de saúde da população. No fio dos discursos dos gestores evidencia-se a desvalorização da problemática da TB tendo em vista, talvez, o desconhecimento da vulnerabilidadesocial das pessoas em adquirir a doença bem como das metas preconizadas pela OM S. A posição dos gestores remeteuma visão epidemiológica assentada no conceito de risco em detrimento ao de vulnerabilidade, essa mais condizente com a construção de espaços de cogestão e na produção de cuidado em saúde enquanto valor e respeito à cidadania.

O desenvolvimento das práticas e ações de saúde voltadas para o conceito de risco constroem representações não condizentes à complexidade dos processos, ou seja, não são capazes de desenhar os interesses, os val ores e as necessidades, seja no campo individual ou coletivo, deuma determinada sociedade. Esteconceito contribuiu para a produção de determinadas racionalidades, estratégias esubjetividades, sendo central na regulação e no monitoramento de indivíduos, grupos sociais e instituições ${ }^{22}$.

As práticas de cuidado assentadas na vulnerabilidade devem incorporar o contexto territorial, na medida em queeste podeacarretar maior suscetibilidade à infecção e ao adoecimento e, de modo inseparável, à maior ou menor disponibilidade de recursos de todas as ordens para a proteção das pessoas contra as doenças ${ }^{23}$.

Em relação ao planejamento em saúde, para gestão do cuidado à TB, tem se mostrado enraizado na normatização de suas ações. Embora se reconheça todo esforço da gestão e de trabal hadores de saúde, principalmente nos níveis centrais das Secretarias; a prática do planejamento para as ações de controledaTB, ainda, não confi- 
gura uma prioridade nos serviços de saúde. Poucos são os trabal hos que propõem e/ou adotam novas estratégias para realização do planejamento da TB nos moldes do pensamento estratégico situacional (PES).

O PES visa à compreensão do objeto plane jado como sendo os problemas e as oportunidades reais de intervenção, possibilita uma articulação das experiências acumuladas nas distintas práticas das várias instituições envolvidas com a política de saúde; flexibiliza a organização e facilita a participação de distintos sujeitos sociais e; finalmente, potencializa o processo de democratização das relações euma intensificação das ações comunicativas entre dirigentes, técnicos eusuários dos serviços ${ }^{24}$. N essa compreensão existem três barreiras a serem ultrapassadas: a primeira refere-se à inserção da prática do planejamento nos diversos serviços de saúde; a segunda a ruptura com a forma normativa da construção do planejamento e a terceira de inserir na agenda; não apenas da política, dos pactos e dos planos; mas na agen da dos serviços de atenção básica, principalmente das equipes de SF, a TB como prioridade no planejamento de suas ações na linha do pensamento estratégico situacional.

Para elaborar estratégias de controle da TB, é preciso planejar as ações de modo a promover a integralidade, a intersetorialidade e a interdisciplinaridade, na tentativa de determinar ações que propiciem a cura e a reinserção do indivíduo, doente de TB, na sociedade ${ }^{25}$. É preciso compreender o planejamento como uma ferramenta de gestão que permite que os atores das instituições realizem seu trabalho em função de objetivos claros e explícitos, do mesmo modo que os gestores poderão reconhecer e acompanhar o trabalho dos que se encontram sob a sua orientação 24,25 . Desse modo, existe a necessidade de instigar a cultura do planejamento nos serviços de saúde, não o planejamento normativo, conforme revelam os discursos de alguns gestores, mas um planejamento que possibilite a construção de um projeto coletivo e de uma perspectiva de realiza- ção profissional, em vista a ampliar a capacidade operativa do coletivo ${ }^{25}$.

Em síntese, as práticas de planejamento para a TB são fundamentais tanto para a organização dos serviços, de modo a possibilitar a detecção precoce e o tratamento da doença, quanto na construção de espaços de cogestão que garantam a exequibilidade e a eficácia da gestão do cuidado à TB.

\section{Conclusões}

A efetivação da gestão do cuidado à TB ainda permanece como um dos desafios para a gestão em saúde do município estudado. Fica evidente, nos discursos dos AM, fragilidades conceituais, tanto no que rege a política local, quanto da organização dos serviços de saúde que aponte para o fortal ecimento da gestão do cuidado à TB, articulado no que se baseia o plano de saúde local.

Sugere-se que a gestão dispare processos de discussão e reflexão sobre a gestão do cuidado junto aos AM , uma vez que exercem atividades gerenciais. Primeiramente, sobre os processos instituintes aos quais estão envolvidos, que devem perpassar a ruptura de práticas hegemônicas instituídas e de saberes reducionistas do processo saúde- doença-cuidado para práticas democráticas, de inclusão ede solidariedade. Segundo, na perspectiva da cogestão, a TB deve ser compreendida não apenas como uma doença, mas como problema mundial de saúde que precisa ser enfrentado nos aspectos de vulnerabilidade social, do estigma, da subjetividade e de singularidade dos sujeitos envolvidos.

Nesse sentido, a gestão do cuidado à $T B$, considerando o compromisso político dos gestores, além de assegurar a atenção de qualidade nos serviços de saúde e de qualificar AM, deve criar e ou implementar ações que ampliem a participação do usuário nas rodas de discussão que envolvam planejamento e ações voltadas ao controle da TB. 


\section{Colaboradores}

AJR Barrêto, LD Sá, JA Nogueira, PF Palha, PGOD Pinheiro, TCS Villa participaram igualmente na elaboração do projeto, coleta de dados, análise eelaboração do texto do artigo. N M P Farias, DCS Rodrigues participaram no desenvolvimento do projeto, coleta e análise do material empírico.

\section{Referências}

1. Almeida PF, Fausto MCR, Giovanella L. Fortalecimento da atenção primária à saúde: estratégias para poencializar a coordenação dos cuidados. Rev Panam Salud Publica 2011; 29(2):84-95.

2. Hijjar MA, Gerhardt G, Teixeira GM e Procópio MJ. Retrospecto do controle da tuberculose no Brasil. Rev Saude Publica 2010; 41(Supl.1):50-58.

3. Dye C. Tuberculosis 2000-2010: control, but not elimination. Int J.Tuberc. Lung Dis. 2000; 4(Supl. 2):146-152.

4. Pires MRGM, Göttems LBD. Análise da gestão do cuidado no Programa de Saúde da Família: referencial teórico-metodológico. Rev. bras. enferm. 2009; 62(2):294-299.

5. Cecílio LCO. The death of Ivan Ilyich, by Leo Tolstoy: points to be considered regarding the multiple dimensions of healthcare management. Interface C 0 mun Saude Educ 2009; 13(Supl.1):545-555.

6. João Pessoa. Secretaria M unicipal de Saúde (SMS). Plano M unicipal de Saúde. 2006-2009. João Pessoa (PB): SM S; 2006.

7. Orlandi EP. Análise de Discurso: princípios e procedimentos. 8a ed. Campinas (SP): Pontes; 2009.

8. Lima DS. Documento técnico contendo dados consolidados dos principais indicadores que caracterizam a situação da tuberculose dos municípios prioritários (João Pessoa, Patos e Santa Rita) do estado da Paraíba. 2008. OPAS. Secretaria de Estado da Saúde da Paraíba. Paraíba: Gerência Executiva de Vigilância em saúde, Núcleo de Doenças Endêmicas, Mar, Paraíba, 2008.

9. Palha PF, Villa TCS. A descentralização como eixo norteador na reorganização e operacionalização dos princípios do Sistema Único de Saúde. Rev. esc. enferm. USP 2003; 37(3):19-26.

10. Giovanella L, M endonça M HM, Almeida PF, Escorel S, Senna MCM, Fausto MCR, Delgado MM, Andrade CLT, Cunha MS, Martins MIC e Teixeira CP. Saúde da família: limites e possibilidades para uma abordagem integral de atenção primária à saúde no Brasil. Cien Saude Colet 2009; 14(3):783-794.

11. Sá LD, Oliveira AAV, Souza KMJ, Palha PF, Nogueira JA e Villa TCS. Abandono do tratamento e elenco de serviços no cuidado ao doente de tuberculose. Rev. enfermagem. UFPE 2010; 4(3):178-186.

12. Oliveira GN. Apoio matricial como tecnologia de gestão e articulação em rede. In: Campos GWS, Guerreiro AVP, organizadores. Manual de práticas de Atenção Básica: saúde ampliada e compartilhada. 2a ed. São Paulo: Aderaldo \& Rothschild; 2010. p. $273-282$.

13. Campos GWS. Um método para análise e co-gestão de coletivos: a constituição do sujeito, a produção de valor de uso e a democracia em instituições: 0 método da roda. São Paulo: Hucitec; 2000.

14. Nogueira JA, Sá LD, França UM, Almeida AS, Lima DS, Figueiredo TMRM, Villa TCS. O sistema de informação e o controle da tuberculose nos municípios prioritários da Paraíba - Brasil. Rev. esc. enferm. USP 2009; 43(1):125-131.

15. Word Health Organization (WHO). Global tuberculosis control: surveillance, planning, financing. In: Brazil: country profile. Geneva: WHO; 2006. p. 77-79. 
16. Terra M F, Bertolozzi MR. Tratamento Diretamente Supervisionado (DOTS) contribui para adesão ao tratamento da tuberculose? Rev. Latino-am Enfermagem 2008; 16(4):659-664.

17. Brasil. M inistério da Saúde. Secretaria de Vigilância em Saúde. Programa Nacional de Controle da Tuberculose. M anual de Recomendações para o Controle da Tuberculose no Brasil. [acessado em 2010 out 19]. 2010. Disponível em: http://portal.saude.gov.br/ portal/arquivos/pdf/manual_de_recomendacoes controle tb novo.pdf.

18. Formiga NS, Lima DS. A tuberculose no Estado da Paraíba/Brasil: a operacionalidade do tratamento supervisionado no controle da tuberculose. Conscientiae Saúde 2009; 8(2):197-201.

19. M erhy EE. Saúde: a cartografia do trabalho vivo. $3^{3}$ ed. São Paulo: Hucitec; 2007.

20. Ayres JRCM. Sujeito, intersubjetividade e práticas de saúde. Cien Saude Colet 2001; 6(1):63-72.

21. Barros S, Oliveira MAF, Silva ALA. Práticas inovadoras para o cuidado em saúde. Rev. esc. enf. USP 2005; 41(Esp):815-819.

22. Czeresnia D. O Conceito de Saúde e a Diferença entre Prevenção e Promoção. In: Czeresnia D, organizador. Promoção da saúde: conceitos, reflexões, tendências. Rio de Janeiro: Fiocruz; 2003. p. 39-53.

23. Ayres JRCM, Calazans GJ, Saletti Filho HC e França-Júnior I. Risco, vulnerabilidade e práticas de prevenção e promoção da saúde. In: Campos GWS, M inayo MCS, Akerman M, Drumond Júnior $M$, Carvalho YM, organizadores. Tratado de Saúde Coletiva. São Paulo, Rio de Janeiro: Hucitec, Fiocruz; 2006. p.375-417.

24. Teixeira CF, Jesus WLA. Correntes de pensamento em planejamento de saúde no Brasil. In: Teixeira $C F$, organizador. Planejamento em saúde: conceitos, métodos e experiências. Salvador: EDUFBA; 2010. p. 33-50.

25. Paim JS. Planejamento em Saúde para não Especialistas. In.: Campos GWS, M inayo MCS, Akerman $M$, Drumond Júnior $M$, Carvalho YM. Tratado de Saúde Coletiva. São Paulo, Rio de Janeiro: Hucitec, Fiocruz; 2007. p. 767-782.

Artigo apresentado em 17/04/2011

Aprovado em 11/05/2011

Versão final apresentada em 01/08/2011 